\title{
Composto orgânico à base de salvínia para a produção de mudas de grandiúva
}

Nereu Carvalho de Sousa ${ }^{1}$ (iD, Bruno Lisboa 2 (iD), Luciano Kayser Vargas² (iD) Sérgio Augusto de Loreto Bordignon ${ }^{3}$ (iD, Anelise Beneduzi ${ }^{*}(\mathbb{D})$

${ }^{1}$ Fundação Zoobotânica do Rio Grande do Sul, Rua Dr. Salvador França, 1427, CEP 90690-000, Porto Alegre, RS, Brasil

${ }^{2}$ Secretaria da Agricultura, Pecuária e Desenvolvimento Rural, Rua Gonçalves Dias, 570, CEP 90130-060, Porto Alegre, RS, Brasil

${ }^{3}$ Universidade La Salle, Avenida Victor Barreto, 2288, CEP 92010-000, Canoas, RS, Brasil

*Autor correspondente:

anebeneduzi@gmail.com

Termos para indexação:

Adubação orgânica

Substrato

Trema micrantha

Index terms:

Organic fertilization

Substrate

Trema micrantha

Histórico do artigo:

Recebido em 17/10/2018

Aprovado em 22/05/2020

Publicado em 12/02/2021

\begin{abstract}
Resumo - Objetivou-se avaliar a eficiência de proporções de salvínia na composição de substrato para a produção de mudas de grandiúva. As características químicas do substrato foram melhoradas à medida que a proporção aumentou, resultando em uma redução de $\mathrm{Al}$ e aumento de $\mathrm{pH}, \mathrm{CTC}$, saturação de bases, teores de $\mathrm{K}, \mathrm{Ca}, \mathrm{Mg}, \mathrm{P}$ e matéria orgânica. $\mathrm{O}$ crescimento das mudas de grandiúva foi influenciado pelos níveis de fertilidade do substrato. As maiores alturas de planta, diâmetros de caule, massa seca e acúmulo de macronutrientes foram verificadas nos tratamentos com $75 \%$ e $100 \%$ de composto orgânico.
\end{abstract}

\section{Organic compound based on salvinia to grow Jamaican nettletree seedlings}

\begin{abstract}
This study aimed to evaluate the efficiency of salvinia proportions in the substrate composition to grow Jamaican nettletree seedlings. The chemical characteristics of the substrate were improved as the dosage increased, resulting in $\mathrm{Al}$ reduction and increased $\mathrm{pH}, \mathrm{CTC}$, base saturation, $\mathrm{K}, \mathrm{Ca}, \mathrm{Mg}, \mathrm{P}$ and organic matter contents. Growth of Jamaican nettletree seedlings was influenced by the fertility levels of the substrate. The highest plant height, stem diameter, dry mass, and macronutrient accumulation were verified in treatments with $75 \%$ and $100 \%$ of the organic compound.
\end{abstract}

Trema micrantha (L.), comumente conhecida como grandiúva, possui uma ampla distribuição desde o sul da Flórida e México até o norte da Argentina (Freitas et al., 2016). Em território brasileiro concentra-se especialmente no Bioma Mata Atlântica (Stolarski et al., 2018). É uma arbórea perenifólia e uma das primeiras espécies que ocorrem em áreas degradadas, existindo em todos os estágios da sucessão secundária, exceto na floresta clímax (Lorenzi, 2008). Devido a isso, ela é muito utilizada para revegetação (Castellani \& Aguiar, 1998). Apesar de sua importância, a utilização da grandiúva, assim como a de muitas espécies nativas, é limitada pela oferta de mudas. A produção de mudas de boa qualidade, vigorosas, com folhas de tamanho e coloração típicas da espécie e em bom estado nutricional (Cruz et al., 2006), e de baixo custo, é condição indispensável para o sucesso de qualquer recomposição de florestas nativas (Fonseca et al., 2002). Assim, a escolha de um substrato deve ser criteriosa. Deve-se levar em conta o tipo de solo, a nutrição e a fisiologia vegetal, buscando combinar as propriedades necessárias para um melhor desenvolvimento da planta. Com a finalidade de reduzir 
custos, minimizar o impacto ambiental e melhorar a qualidade dos substratos, diversos tipos de compostos orgânicos vêm sendo utilizados e reaproveitados em mistura ou em substituição a solos (Meneghelli et al., 2017).

As pteridófitas do gênero Salvinia, nativas da América do Sul e abundantes no Brasil, possuem uma elevada taxa de crescimento, sendo infestantes agressivos de corpos hídricos lênticos, o que causa problemas pelo acúmulo excessivo de massa vegetativa na superfície da água (Mora-Olivo \& Yatskievych, 2009). São uma fonte promissora de compostos orgânicos para a formulação de substratos, por possuírem uma grande capacidade de absorção e retenção de nutrientes (Farias et al., 2013), sendo por esse motivo utilizadas para a remediação de corpos hídricos eutrofizados (Zhou et al., 2017).

A compostagem de resíduos orgânicos resultantes da manutenção do Jardim Botânico (JB) de Porto Alegre, RS, é uma prática sistemática desde a sua fundação em 1975. A partir de 2003, quando foi feita a revitalização de um lago com área aproximada de $7.800 \mathrm{~m}^{2}$, houve uma proliferação excessiva da macrófita aquática Salvinia sp. As macrófitas removidas do lago passaram a ser usadas na produção de um composto orgânico, a partir da mistura de triturados de restos de poda e esterco equino, além de Salvinia sp. Esse composto passou a ser utilizado na produção de mudas, na jardinagem, na manutenção das coleções envazadas e arbóreas ex situ do JB. Considerando-se a necessidade de caracterização de parâmetros físico-químicos de um composto orgânico, bem como a validação experimental da eficiência agronômica para sua utilização como substrato, o presente estudo teve como objetivo avaliar o uso deste composto produzido a partir de Salvinia sp., em diferentes dosagens, em mistura com Argissolo Vermelho-Amarelo distrófico, para a produção de mudas de grandiúva.

O cultivo experimental de mudas de grandiúva foi conduzido no período de janeiro a setembro de 2014, em casa de vegetação do JB (3003'06.07' S, 51 10'37.95” W), pertencente a Fundação Zoobotânica do Rio Grande do Sul. As mudas foram obtidas em uma área de fração florestal do JB e foi possível selecionar um lote uniforme com raízes nuas, aptas para o transplante e com altura média entre 3 e $5 \mathrm{~cm}$.

O composto orgânico utilizado foi produzido a partir da compostagem de Salvinia sp. (80\%, v/v), coletada em um açude do JB, juntamente com esterco equino $(20 \%, \mathrm{v} / \mathrm{v})$ (Tabela 1). A pilha de compostagem foi mantida por um período de quatro meses, sendo o material revolvido três vezes durante o processo e, posteriormente, peneirado em malha $1,0 \mathrm{~cm}^{2}$. Para avaliar a eficiência agronômica do composto orgânico na produção de mudas de grandiúva, foram testadas proporções crescentes $(0,25,50,75$ e $100 \%, \mathrm{v} / \mathrm{v})$ em mistura com um solo Argissolo Vermelho-Amarelo distrófico (Santos et al., 2014) também coletado na área do JB (Tabela 1).

Tabela 1. Composição química do composto orgânico e do solo utilizado para a produção de mudas de grandiúva.

Table 1. Chemical composition of the organic compost and the soil used to grow Jamaican nettletree seedlings.

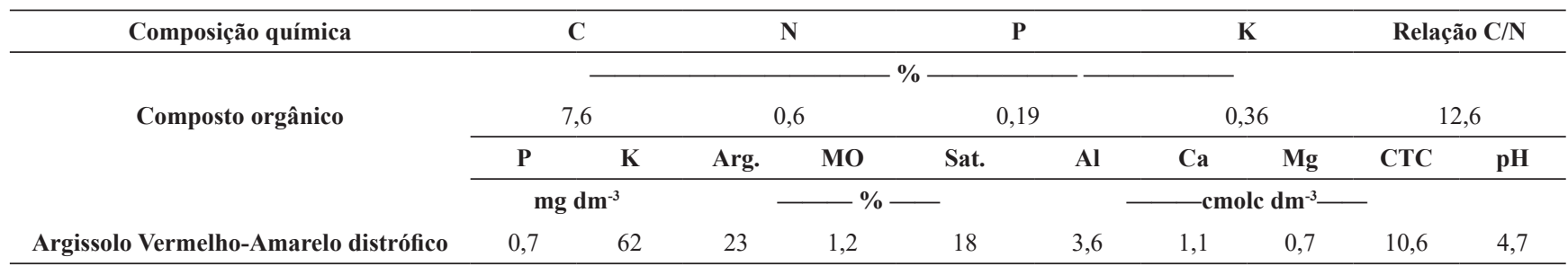

Arg. = teor de argila; $\mathrm{MO}$ = matéria orgânica; Sat. = saturação de bases; $\mathrm{CTC}=$ capacidade de troca de cátions.

O delineamento utilizado foi inteiramente casualizado, com cinco tratamentos e 30 unidades amostrais (sacos de polietileno com capacidade para $2.000 \mathrm{~cm}^{3}$ de substrato) para cada tratamento. $\mathrm{O}$ período de crescimento das mudas foi de sete meses. Ao final, foram avaliados os parâmetros químicos dos substratos e os teores e o conteúdo de N, P e K no tecido vegetal (Tedesco et al., 1995). Avaliou-se também a altura do colo até a gema apical, o diâmetro do caule a $2 \mathrm{~cm}$ do colo e a massa seca total da parte aérea. Os dados relacionados aos parâmetros químicos do substrato de cultivo foram ajustados em equações de regressão com uso do 
programa SigmaPlot 11.0. Os parâmetros de crescimento das mudas foram submetidos à análise de variância (teste F) e as médias foram comparadas com o teste de Tukey a $5 \%$ de probabilidade.

Observou-se uma melhora expressiva das características químicas dos substratos à medida que aumentou a proporção de composto orgânico nas misturas (Figura 1).

O solo utilizado no experimento, característico da Região Metropolitana de Porto Alegre, apresenta baixa fertilidade natural (Tabela 1), enquanto a compostagem de resíduos orgânicos pode resultar em produtos ricos em nutrientes (Carlesso et al., 2011).
O aumento da proporção de composto orgânico no substrato levou a um aumento linear do $\mathrm{pH}$, a partir de 4,7 ( $0 \%$ de composto) até 6,4 (100\% de composto) (Figura 1a). Um comportamento semelhante também foi observado por Krob et al. (2011), avaliando em campo o impacto da aplicação de um composto orgânico de lixo urbano sobre as propriedades químicas de um Argissolo Vermelho. Os autores atribuíram a elevação do $\mathrm{pH}$ à maior mineralização de matéria orgânica, com produção subsequente de íons $\mathrm{OH}^{-}$, e à introdução de

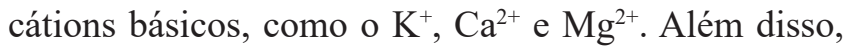
a elevação do $\mathrm{pH}$ no substrato de cultivo também pode estar relacionada à redução da atividade de $\mathrm{H}^{+}$pela a)

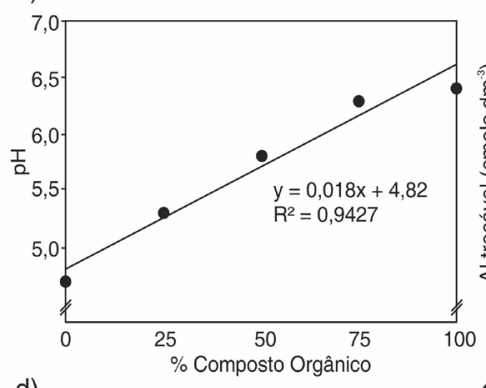

d)

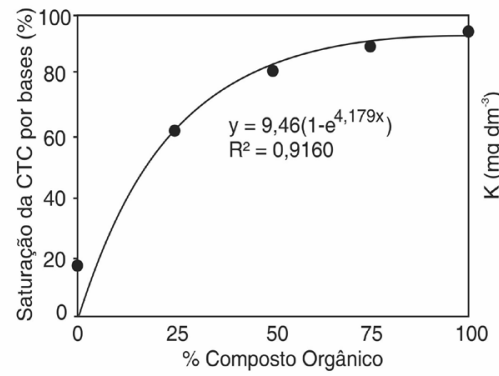

g)

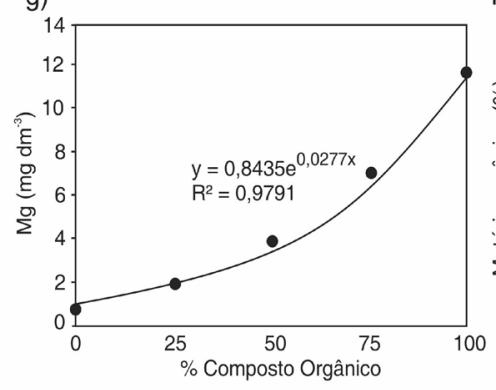

b)

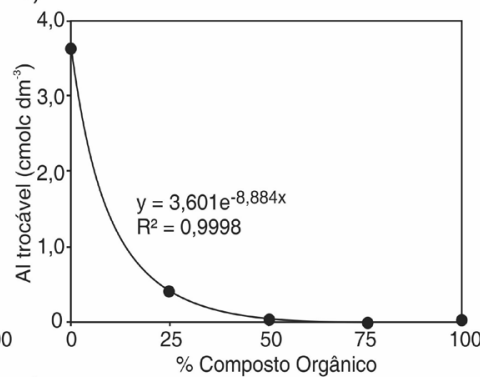

e)

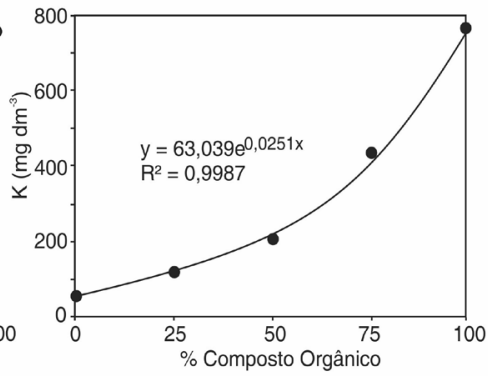

h)

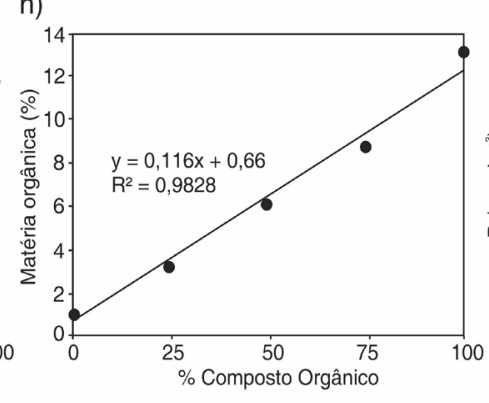

c)
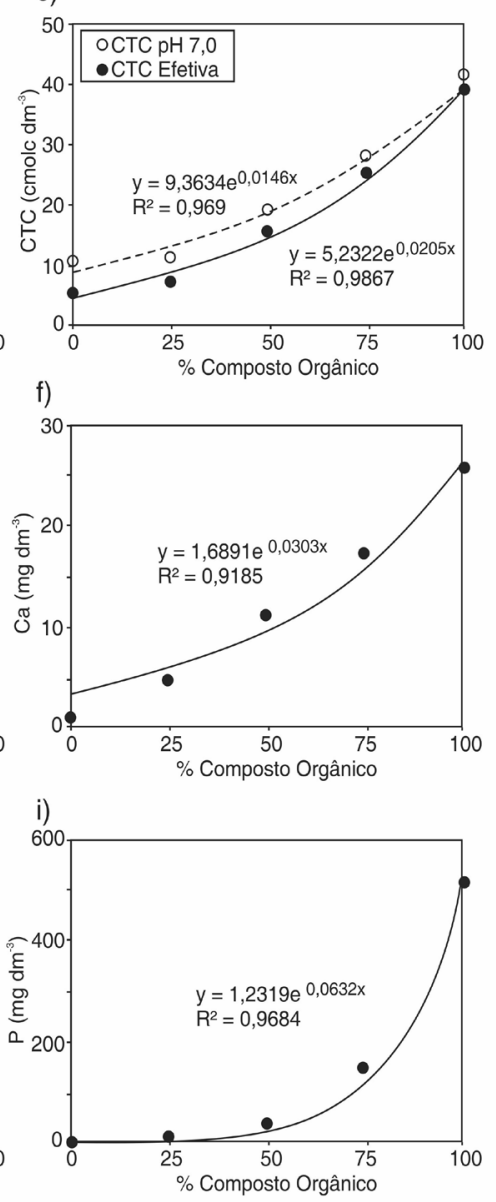

Figura 1. Características químicas dos substratos com proporções crescentes do composto orgânico à base de salvínia para a produção de mudas de grandiúva.

Figure 1. Chemical composition of the substrate using increasing proportions of organic compost based in salvinia used to grow Jamaican nettletree seedlings. 
liberação de cátions metálicos, com a mineralização de formas orgânicas de $\mathrm{N}$, com a desnitrificação e com a descarboxilação de ácidos orgânicos (Silva \& Mendonça, 2007).

A elevação do $\mathrm{pH}$ no substrato de cultivo, em decorrência da maior concentração do composto orgânico, foi acompanhada por um decréscimo do teor de $\mathrm{Al}$ trocável (Figura $1 \mathrm{~b}$ ). $\mathrm{O}$ teor de $\mathrm{Al}^{3+}$ no tratamento contendo apenas o solo base ( $0 \%$ de composto) foi de $3,6 \mathrm{cmolc} \mathrm{dm}^{-3}$, com pH 4,7. Com a concentração de $25 \%$ de composto orgânico e pH 5,3 , o teor de $\mathrm{Al}^{3+}$ caiu para $0,4 \mathrm{cmol}_{\mathrm{c}} \mathrm{dm}^{-3}$, não sendo detectado a partir da concentração de $50 \%$ de composto orgânico $(\mathrm{pH}=5,8)$. Este comportamento é esperado, tendo em vista que a solubilidade do $\mathrm{Al}$ diminui com o aumento de $\mathrm{pH}$, no solo ou no substrato, e, quando o $\mathrm{pH}$ do solo atinge valores superiores a 5,4 - 5,5, o $\mathrm{Al}^{3+}$ precipita completamente (Ernani et al., 2000). Além do aumento do $\mathrm{pH}$ do substrato de cultivo, a redução dos teores de $\mathrm{Al}^{3+}$ nas maiores proporções de composto orgânico pode se dar também pela complexação do cátion pela matéria orgânica, principalmente por ácidos fúlvicos, formando complexos de esfera interna, reduzindo a disponibilidade do Al trocável (Sposito, 2008).

A capacidade de troca de cátions (CTC) do substrato de cultivo também foi afetada pelas proporções de composto orgânico (Figura 1c). Tanto a CTC efetiva $\left(\mathrm{CTC}_{\text {ef. }}\right)$ quanto a pH 7,0 $\left(\mathrm{CTC}_{\mathrm{pH} 7}\right)$ apresentaram aumento exponencial com a adição de composto. Com apenas o solo como base do substrato de cultivo, a diferença da CTC efetiva para a potencial era de aproximadamente $100 \%$, enquanto no tratamento contendo apenas composto orgânico a $\mathrm{CTC}_{\text {ef. }}$ tornou-se igual à potencial. As $\mathrm{CTC}_{\mathrm{pH} 7,0}$ observadas nos tratamentos com 0 e $25 \%$ de composto orgânico (10,6 e 11,5 cmolc dm ${ }^{-3}$, respectivamente) são consideradas médias, pela recomendação oficial dos estados do Rio Grande do Sul e Santa Catarina (CQFS$\mathrm{RS} / \mathrm{SC}, 2016)$. A partir de $50 \%$ de composto orgânico $\left(19,1 \mathrm{cmol}_{\mathrm{c}} \mathrm{dm}^{-3}\right)$, os valores foram considerados altos, chegando a $41,48 \mathrm{cmol}_{\mathrm{c}} \mathrm{dm}^{-3}$ com $100 \%$ de composto orgânico.

Frequentemente, o aumento da CTC de um solo ou substrato está associado ao aumento de matéria orgânica e pH (Dorneles et al., 2015). O aumento de $\mathrm{pH}$ do substrato de cultivo leva ao incremento de cargas negativas na matéria orgânica, principalmente de sua fração humificada, a partir da dissociação de radicais carboxílicos, sendo este incremento linear entre o $\mathrm{pH}$
3 e 6,5 (Silva \& Mendonça, 2007). De acordo com Sposito (2008), este comportamento faz com que a matéria orgânica humificada apresente cargas negativas a partir de valores baixos de $\mathrm{pH}(4,0)$, sendo que a sua interação com argilominerais $1: 1$, como a caulinita (predominante nos solos tropicais), faz com que o $\mathrm{pH}$ do ponto de carga zero (PCZ) do solo/substrato seja menor, e por consequência, aumente a CTC. O mesmo autor complementa que este efeito é potencializado pela elevada superfície específica da matéria orgânica. O aumento da CTC da matéria orgânica, com a elevação do $\mathrm{pH}$, faz com que o poder tampão do substrato de cultivo também aumente, tendo em vista que a maior densidade de cargas negativas, além de reter mais cátions metálicos, também neutraliza mais prótons. Dentro do conceito fonte/dreno, um solo ou substrato com CTC elevada tem maior potencial para fornecer nutrientes cationicos para as plantas, principalmente aqueles demandados em maiores quantidades, como $\mathrm{Ca}, \mathrm{Mg}$ e $\mathrm{K}$, pois ocorrerá a reposição satisfatória destes a partir dos pontos de troca (fonte) para solução do solo, mesmo com o aumento da taxa de absorção pelas raízes (dreno).

Neste contexto, a saturação por bases (SB) também apresentou comportamento semelhante, havendo um crescimento assintótico, estabilizando-se em 94\% na proporção mais elevada de composto orgânico (Figura 1d). A SB é uma propriedade relacionada com a CTC e também com os teores de cátions trocáveis presentes no solo ou substrato. Além disso, o incremento da SB também está relacionado com a elevação do pH do substrato de cultivo. Este acarretou a redução da participação do $\mathrm{Al}^{3+}$ no complexo sortivo e, por consequência, disponibilizou sítios para serem ocupados por cátions básicos, resultando em um incremento exponencial dos teores das bases trocáveis $\mathrm{K}, \mathrm{Ca}$ e $\mathrm{Mg}$ (Figuras 1e, 1f e 1g, respectivamente).

A interpretação dos níveis de $\mathrm{K}^{+}$nos diferentes tratamentos é determinada pela $\mathrm{CTC}_{\mathrm{pH} 7}$ observada em cada situação (CQFS-RS/SC, 2016). Dessa forma, os teores de $\mathrm{K}$ trocável no substrato de cultivo constituído apenas com solo base $\left(62 \mathrm{mg} \mathrm{dm}^{-3} ; \mathrm{CTC}_{\mathrm{pH} 7}=\right.$ $10,6 \mathrm{cmol}_{\mathrm{c}} \mathrm{dm}^{-3}$ ) e no tratamento com $25 \%$ de composto orgânico (122 mg DM"-3 $\left.\mathrm{CTC}_{\mathrm{pH} 7}=11,5 \mathrm{cmol}_{\mathrm{c}} \mathrm{dm}^{-3}\right)$, estão enquadrados como altos. Nos demais tratamentos, com $50 \%, 75 \%$ e $100 \%$ de composto orgânico, os teores de $\mathrm{K}^{+}$de $213\left(\mathrm{CTC}_{\mathrm{pH} 7}=19,1 \mathrm{cmol}_{\mathrm{c}} \mathrm{dm}^{-3}\right), 432$ $\left(\mathrm{CTC}_{\mathrm{pH} 7}=28,2 \mathrm{cmol}_{\mathrm{c}} \mathrm{dm}^{-3}\right)$ e $759 \mathrm{mg} \mathrm{dm}{ }^{-3}\left(\mathrm{CTC}_{\mathrm{pH} 7}\right.$ $\left.=41,8 \mathrm{mg} \mathrm{dm}^{-3}\right)$, respectivamente, são considerados 
muito altos, de acordo com a recomendação oficial para os estados do RS e SC. Em relação às demais bases trocáveis, o tratamento contendo apenas solo apresentou nível baixo de $\mathrm{Ca}$ e médio de $\mathrm{Mg}$. Todos os demais tratamentos apresentaram níveis altos de $\mathrm{Ca}$ e $\mathrm{Mg}$, sendo que os seus teores aumentaram de forma exponencial até as concentrações máximas de 25,8 e $11,6 \mathrm{cmol}_{\mathrm{c}} \mathrm{dm}^{-3}$, respectivamente.

A matéria orgânica no substrato de cultivo apresentou crescimento linear, conforme aumentou o teor do composto orgânico, em função do elevado conteúdo de $\mathrm{C}$ do composto (Figura 1h). Este incremento de $\mathrm{C}$ afetou o comportamento dos parâmetros $\mathrm{pH}, \mathrm{CTC}$ e SB. Além disso, o aumento de $\mathrm{C}$ no substrato de cultivo também está acompanhado de um aumento da capacidade deste em fornecer $\mathrm{N}$ para as mudas de grandiúva. A relação $\mathrm{C} / \mathrm{N}$ do composto orgânico à base de Salvinia sp. é baixa $(12,6)$, ainda mais se comparada com outros materiais orgânicos utilizados como substratos, tais como casca de arroz carbonizada $(30,5)$, casca de arroz cru $(50,0)$, fibra de coco verde $(74,1)$, maravalha $(98,9)$ e serragem $(156,5)$ (Carrijo et al., 2004). Esta relação $\mathrm{C} / \mathrm{N}$ do composto é semelhante à de leguminosas (Aita et al., 2001) e situa-se abaixo da relação $\mathrm{C} / \mathrm{N}$ crítica, a qual está entre 20 e 30 e abaixo da qual o processo de mineralização predomina sobre o de imobilização (Vargas et al., 2005), indicando que este composto atua como uma fonte de $\mathrm{N}$ para as plantas.

O teor de P disponível nos substratos de cultivo (Figura 1i) apresentou comportamento exponencial, sendo, inicialmente, considerado muito baixo ( $0 \%$ de composto orgânico), passando para médio ( $25 \%$ de composto orgânico) e muito alto, a partir de $50 \%$ de composto orgânico, chegando a $428 \mathrm{mg} \mathrm{dm}^{-3}$. A elevação do $\mathrm{pH}$, em decorrência da adição do composto orgânico ao substrato $(25,50$ e $75 \%)$, pode ter ocasionado aumento dos teores de $\mathrm{P}$, devido ao deslocamento do nutriente adsorvido na superfície de óxidos de Fe e de Al por hidroxilas (Souza et al., 2006). Entretanto, em função da baixa fertilidade natural do solo utilizado na composição dos diferentes substratos, é pouco provável que o P proveniente do solo tenha contribuído significativamente para o aumento da disponibilidade do nutriente, sendo a mineralização do $\mathrm{P}$ orgânico o fator que mais contribuiu, à medida que a proporção de composto orgânico no substrato aumentou. Nesse contexto, considerando-se que o composto orgânico possuía $0,19 \%$ de $\mathrm{P}$ total no início do experimento e que o teor de $\mathrm{P}$ disponível no tratamento com $100 \%$ de composto orgânico foi de $428 \mathrm{mg} \mathrm{dm}^{-3}$, conclui-se que praticamente $30 \%$ do P orgânico original foi mineralizado, passando a ser disponível para as mudas de grandiúva.

A partir da análise dos parâmetros químicos, notase que os substratos de cultivo com 50, 75 e $100 \%$ de composto orgânico apresentaram os melhores níveis de fertilidade. Assim, determinou-se a altura e diâmetro do caule das plantas, bem como o acúmulo de massa seca na parte aérea, visando à avaliação da eficiência agronômica dos diferentes substratos de cultivo na produção de mudas de grandiúva. Neste contexto, a resposta das mudas acompanhou o aumento dos níveis de composto orgânico nos substratos (Tabela 2). A altura das mudas aumentou significativamente a cada incremento da dosagem do composto orgânico até $75 \%$ e $100 \%$. O mesmo comportamento foi observado com relação ao diâmetro do caule e ao acúmulo de massa seca das mudas, os quais também foram superiores nos tratamentos com 75 e 100\%. Deve-se salientar ainda que a massa seca das mudas produzidas com $75 \%$ e $100 \%$ do composto à base de salvínia excedeu em mais de vinte vezes ao do tratamento controle de $0 \%$.

Tabela 2. Características das mudas de grandiúva crescidas em substratos com proporções crescentes do composto orgânico à base de salvínia.

Table 2. Characteristics of Jamaican nettletree seedlings growing in substrate using increasing proportions of organic compost based in salvinia.

\begin{tabular}{cccccc}
\hline $\begin{array}{c}\text { Característica da } \\
\text { muda }\end{array}$ & \multicolumn{5}{c}{ Proporção de composto orgânico no substrato } \\
\cline { 2 - 6 } & $\mathbf{0}$ & $\mathbf{2 5 \%}$ & $\mathbf{5 0 \%}$ & $\mathbf{7 5 \%}$ & $\mathbf{1 0 0 \%}$ \\
\hline Altura (cm) & $3,86 \mathrm{~d}$ & $10,38 \mathrm{c}$ & $20,13 \mathrm{~b}$ & $27,23 \mathrm{a}$ & $31,18 \mathrm{a}$ \\
$\begin{array}{c}\text { Diâmetro do } \\
\text { coleto (mm) }\end{array}$ & $1,91 \mathrm{~d}$ & $3,13 \mathrm{c}$ & $4,48 \mathrm{~b}$ & $5,6 \mathrm{a}$ & $5,8 \mathrm{a}$ \\
$\begin{array}{c}\text { Massa seca } \\
\text { total (g) }\end{array}$ & $0,17 \mathrm{~d}$ & $1,22 \mathrm{c}$ & $3,12 \mathrm{~b}$ & $3,73 \mathrm{a}$ & $4,87 \mathrm{a}$ \\
\hline
\end{tabular}

Médias seguidas por letras distintas, na linha, diferem entre si pelo teste de Tukey ao nível de 5\% de significância.

O teor de macronutrientes, como o $\mathrm{N}$, foi significativamente maior no substrato com $100 \%$ de composto orgânico (Tabela 3). Este tratamento também apresentou as maiores quantidades de $\mathrm{P}$ e de $\mathrm{K}$, porém não diferindo dos tratamentos com 50 e $75 \%$. O substrato contendo apenas solo, por outro lado, apresentou teores significativamente menores de nutrientes do que os tratamentos contendo o composto orgânico. Assim como observado em mudas de gravioleira por Barbosa et al. 
(2003), o acúmulo de macronutrientes acompanhou a produção de massa seca e ocorreu na ordem decrescente $\mathrm{K}>\mathrm{N}>\mathrm{P}$.

Tabela 3. NPK em mudas de grandiúva cultivadas em substratos com proporções crescentes do composto orgânico à base de salvínia.

Table 3. NPK in Jamaican nettletree seedlings growing in substrate using increasing proportions of organic compost based in salvinia.

\begin{tabular}{cccccc}
\hline $\begin{array}{c}\text { Nutrientes na } \\
\text { parte aérea }\end{array}$ & \multicolumn{5}{c}{ Proporção de composto orgânico no substrato (\%) } \\
\cline { 2 - 6 } & $\mathbf{0}$ & $\mathbf{2 5}$ & $\mathbf{5 0}$ & $\mathbf{7 5}$ & $\mathbf{1 0 0}$ \\
\cline { 2 - 6 } & & \multicolumn{5}{c}{$\mathbf{m g} /$ planta } \\
$\mathbf{N}$ & $1,67 \mathrm{c}$ & $18,30 \mathrm{c}$ & $59,98 \mathrm{~b}$ & $57,82 \mathrm{~b}$ & $72,08 \mathrm{a}$ \\
$\mathbf{P}$ & $0,08 \mathrm{c}$ & $5,87 \mathrm{~b}$ & $34,36 \mathrm{a}$ & $41,03 \mathrm{a}$ & $32,14 \mathrm{a}$ \\
$\mathbf{K}$ & $2,06 \mathrm{c}$ & $30,62 \mathrm{bc}$ & $98,72 \mathrm{ab}$ & $113,39 \mathrm{a}$ & $138,79 \mathrm{a}$ \\
\hline
\end{tabular}

Médias seguidas por letras distintas, na linha, diferem entre si pelo teste de Tukey ao nível de 5\% de significância.

\section{Conclusões}

De um modo geral, a presença de compostos orgânicos torna o substrato menos denso e aumenta a disponibilidade de água para as plantas (Xue \& Farrell, 2020), além das alterações nas características químicas observadas neste trabalho. Portanto, a presença de $75 \%$ e $100 \%$ de composto orgânico à base de Salvinia sp. melhorou as condições de fertilidade do substrato em mistura com Argissolo Vermelho-Amarelo distrófico e propiciou a produção de mudas mais vigorosas de grandiúva.

\section{Referências}

Aita, C. et al. Plantas de cobertura de solo como fonte de nitrogênio ao milho. Revista Brasileira de Ciência do Solo, v. 25, n. 1, p. 157-165, 2001.

Barbosa, Z. et al. Crescimento e absorção de nutrientes por mudas de gravioleira. Revista Brasileira de Fruticultura, v. 25, n. 3, p. 519-522, 2003.

Carlesso, W. M. et al. Tratamento de resíduos a partir de compostagem e vermicompostagem. Revista Destaques Acadêmicos, v. 3, n. 4, p. 105-110, 2011.

Carrijo, A. O. et al. Produtividade do tomateiro em diferentes substratos e modelos de casas de vegetação. Horticultura Brasileira, v. 22, n. 1, p. 5-9, 2004.
Castellani, E. D. \& Aguiar, I. B. D. Condições preliminares para a germinação de sementes de candiúba (Trema micrantha (L.) Blume). Revista Brasileira deEngenhariaAgrícola eAmbiental,v. 2,n. 1,p. 8083, 1998. https://doi.org/10.1590/1807-1929/agriambi.v2n1p80-83.

CQFS-RS/SC. Comissão de Química e Fertilidade do Solo dos Estados do Rio Grande do Sul e Santa Catarina. Manual de adubação e calagem para os Estados do Rio Grande do Sul e Santa Catarina. Porto Alegre: Sociedade Brasileira da Ciência do Solo, 2016. $376 \mathrm{p}$.

Cruz, C. A. et al. Efeito da adubação nitrogenada na produção de mudas de sete-cascas (Samanea inopinata (Harms) Ducke). Revista Árvore, v. 30, n. 4, pp. 537-546, 2006. https://doi.org/10.1590/S0100-67622006000400006.

Dorneles, E. P. et al. Tillage, fertilization systems and chemical attributes of a Paleudult. Scientia Agricola, v. 72, n. 2, p. 175-186, 2015. https://doi.org/10.1590/0103-9016-2013-0425.

Ernani, P. R. et al. Influência da combinação de fósforo e calcário no rendimento de milho. Revista Brasileira de Ciência do Solo, v. 24, n. 3, p. 537-544, 2000.

Farias, W. M. et al. Propriedades físicas e químicas de substratos produzidos utilizando macrófitas aquáticas. Semina: Ciências Agrárias, v. 34, n. 34, p. 3257-3270, 2013. DOI: 10.5433/1679-0359.2013v34n6Supl1p3257.

Fonseca, E. D. P. et al. Padrão de qualidade de mudas de Trema micrantha (L.) Blume, produzidas sob diferentes períodos de sombreamento. Revista Árvore, v. 26, n. 4, p. 515-523, 2002.

Freitas, W. K. et al. Floristic, diversity and spatial distribution of tree species in a dry forest in Southern Brazil. Applied Ecology and Environmental Research, v. 15, n. 1, p. 511-524, 2016.

Krob, A. D. et al. Propriedades químicas de um Argissolo tratado sucessivamente com composto de lixo urbano. Ciência Rural, v. 41, n. 3, p. 433-439, 2011.

Lorenzi, H. Árvores brasileiras: manual de identificação e cultivo de plantas arbórea nativas do Brasil. 5 ed. Nova Odessa, SP: Instituto Plantarum, 2008. v. 1.368 p.

Meneghelli, L. A. M. et al. Produção de mudas de café arábica em substrato composto por resíduo da secagem dos grãos. Coffee Science, v. 12, n. 3, p. 381-388, 2017.

Mora-Olivo, A. \& Yatskievych, G. Salvinia molesta in Mexico. American Fern Journal, v. 99, n. 1, p. 56-58, 2009.

Santos, H. G. et al. Sistema brasileiro de classificação de solos. Brasília, DF: Embrapa, 2014. 353 p.

Silva, I. R. \& Mendonça, E. S. Matéria orgânica do solo. In: Novais, R. F. et al. (Ed.). Fertilidade do solo. Viçosa, MG: Sociedade Brasileira de Ciência do Solo, 2007. p. 275-374.

Souza, R. F. D. et al. Calagem e adubação orgânica: influência na adsorção de fósforo em solos. Revista Brasileira de Ciência do Solo, v. 30, n. 6, p. 975-983, 2006.

Sposito, G. The chemistry of soils. New York: Oxford University Press, 2008. 342 p. 
Stolarski, O. C. et al. Trema micrantha (L.) Blume. in plantations for ecological restoration: early development in the Brazilian subtropical forest. Ciência Florestal, v. 28, n. 3, p. 1217-1229, 2018.

Tedesco, J. M. et al. Análise de solo, plantas e outros materiais. Porto Alegre: UFRGS, 1995. 174 p.

Vargas, L. K. et al. Imobilização de nitrogênio em solo cultivado com milho em sucessão à aveia preta nos sistemas plantio direto e convencional. Ciência Rural, v. 35, n. 1, p. 76-83, 2005.
Xue, M. \& Farrell, C. Use of organic wastes to create lightweight green roof substrates with increased plant-available water. Urban Forestry \& Urban Greening, v. 48, p. 126569, 2020.

Zhou, X. et al. Dominating aquatic macrophytes for the removal of nutrients from waterways of the Indian River Lagoon basin, South Florida, USA. Ecological Engineering, v. 101, p. 107-119, 2017. https://doi.org/10.1016/j.ecoleng.2017.01.006. 